\title{
CP violation and quark-lepton complementarity of the neutrino mixing matrix
}

\author{
K. Zhukovsky a , A. A. Davydova \\ Department of Theoretical Physics, Faculty of Physics, M.V. Lomonosov Moscow State University, Moscow 119991, Russia
}

Received: 30 December 2018 / Accepted: 18 April 2019 / Published online: 4 May 2019

(C) The Author(s) 2019

\begin{abstract}
The comparative analysis of the neutrino mixing in the standard, cobimaximal and exponential parameterizations is performed. With the latest November 2018 data the logarithm of the mixing matrix is computed and the exact entries for the exponential matrix are obtained. The factorization of the real rotation and the $\mathrm{CP}$ violation in the exponential form of the mixing matrix is demonstrated. Quark-lepton complementarity hypothesis is reformulated, involving three mixing angles in the framework of the exponential parameterisation of the mixing matrix. It is shown that the cobimaximal parameterization, consistent with recent experimental data on neutrino mixing with the spread $3 \sigma$, can provide exact quark-lepton complementarity based on the data for all three mixing angles. The dependence of the $\mathrm{CP}$ violation degree on the parameterization parameters in the standard and the exponential forms is studied with the help of the Jarlskog invariant.
\end{abstract}

\section{Introduction}

The Standard Model (SM) [1-3] successfully describes wide range of particle physics phenomena and is consistent with major experiments held during the last half a century. Neutrinos were introduced in the SM massless with left chirality. However, the prediction of neutrino oscillations $[4,5]$ and their experimental confirmation yielded the conclusion that neutrinos had finite nonzero mass, much smaller than that of other elementary particles. One of the possible ways to introduce small neutrino mass in the SM consists in the addition of extremely heavy right neutrinos to the Lagrangian function of the model $[6,7]$. These extra heavy neutrinos are scalars in $S U(3) \times S U(2) \times U(1)$ group, their mass is giant, $\sim 10^{16} \mathrm{GeV}$, and their observation in modern experiments is impossible. After the introduction of heavy neutrinos, the resulting mass term determines the $\mathrm{CP}$ violation of

a e-mail: zhukovsk@physics.msu.ru the action in the SM and the violation of the lepton-number conservation law within each generation. The presence of non-diagonal entries in the mass matrix means there is neutrino mixing. The contribution of the extra heavy mass states to the mixing is negligible. A unitary transforms exist, which diagonalizes the neutrino mass matrix and yields the states of neutrino with given mass. The observables are the flavor states of neutrinos $\boldsymbol{v}_{e}, \boldsymbol{v}_{\mu}, \boldsymbol{v}_{\tau}$, which are the linear combinations of neutrino mass states $\boldsymbol{v}_{1}, \boldsymbol{v}_{2}, \boldsymbol{v}_{3}$; the transforms is given by the unitary mixing matrix $\mathbf{U}$ Pontecorvo-MakiNakagawa-Sakata (PMNS) [8]:

$$
\begin{aligned}
\left|v_{\alpha}\right\rangle & =\sum_{i=1,2,3} \mathbf{U}_{\alpha i}^{*}\left|v_{i}\right\rangle, \quad \mathbf{U}_{\alpha i} \equiv\left\langle v_{\alpha} \mid v_{i}\right\rangle, \\
\alpha & =e, \mu, \tau, \quad i=1,2,3,
\end{aligned}
$$

The amplitude of the transition for the given flavor $\alpha$ to the mass state $i$ is proportional to $\left|U_{\alpha i}\right|^{2}$. Neutrino mixing can be parameterized in several ways by different matrices. While the physical result obviously does not depend on the choice of the parameterization, the latter may be more or less useful and convenient for certain studies. In what follows we will consider three generations of Dirac neutrinos. Among many parameterizations, the most common is the standard parameterization matrix:

$$
\begin{aligned}
\mathbf{U}_{\mathrm{st}}= & \left(\begin{array}{ccc}
1 & 0 & 0 \\
0 & c_{23} & s_{23} \\
0 & -s_{23} & c_{23}
\end{array}\right)\left(\begin{array}{ccc}
c_{13} & 0 & s_{13} e^{-i \delta_{C P}} \\
0 & 1 & 0 \\
-s_{13} e^{i \delta_{C P}} & 0 & c_{13}
\end{array}\right) \\
& \times\left(\begin{array}{ccc}
c_{12} & s_{12} & 0 \\
-s_{12} & c_{12} & 0 \\
0 & 0 & 1
\end{array}\right) \\
= & \left(\begin{array}{ccc}
-s_{12} c_{23}-c_{12} s_{23} s_{13} e^{i \delta_{C P}} & c_{12} c_{23}-s_{12} s_{23} s_{13} e^{i \delta_{C P}} & s_{13} e^{-i \delta_{C P}} \\
s_{12} s_{23}-c_{12} c_{23} s_{13} e^{i \delta_{C P}} & -c_{12} s_{23}-s_{12} c_{23} s_{13} e^{i \delta_{C P}} & c_{23} c_{13}
\end{array}\right),
\end{aligned}
$$

where $c_{i j}=\cos \theta_{i j}, s_{i j}=\sin \theta_{i j}, i=1,2,3, \theta_{i j}$ are the mixing angles, and $\delta_{C P}$ is the phase, describing the $\mathrm{CP}$ violation. There can be other 11 matrices constructed similarly to (2); 
they differ from each by the complex phase placement and by the order of matrix product (see [9]). It follows from (2) that the $\mathrm{CP}$ violation in $\nu_{\mathrm{e}}$ flavor comes from $\nu_{3}$ mass state; the CP violation in $\nu_{\mu, \tau}$ flavor states is due to the mixed $\nu_{1,2}$ mass states.

In search for symmetries in neutrino mixing, other than (2) parameterizations were proposed. The simplest of them were the bimaximal (BM) and the tribimaximal (TBM) [1013] parameterizations with the following matrices:

$$
\begin{aligned}
\mathbf{U}_{B M} & =\left(\begin{array}{ccc}
\frac{1}{\sqrt{2}} & \frac{1}{\sqrt{2}} & 0 \\
-\frac{1}{2} & \frac{1}{2} & \frac{1}{\sqrt{2}} \\
\frac{1}{2} & -\frac{1}{2} & \frac{1}{\sqrt{2}}
\end{array}\right), \\
\mathbf{U}_{T B M} & =\left(\begin{array}{ccc}
\sqrt{\frac{2}{3}} & \frac{1}{\sqrt{3}} & 0 \\
-\frac{1}{\sqrt{6}} & \frac{1}{\sqrt{3}} & \frac{1}{\sqrt{2}} \\
\frac{1}{\sqrt{6}} & -\frac{1}{\sqrt{3}} & \frac{1}{\sqrt{2}}
\end{array}\right) .
\end{aligned}
$$

The tribimaximal mixing follows from the standard parameterization for $\theta_{13}=0, \theta_{23}=\pi / 4, \tan ^{2} \theta_{12}=1 / 2$. In papers [14-23] it was pointed out that discrete or continuous flavor groups appear to be particularly suitable to reproduce this specific mixing pattern as a first approximation. While the BM and TBM matrices do possess symmetry, they do not describe the $\mathrm{CP}$ violation; moreover, the $\mathrm{BM}$ parameterization $\mathbf{U}_{B M}$ disagrees with contemporary experimental data and the TBM matrix $\mathbf{U}_{T B M}$ (3) just vaguely agrees with the experiments (see [13]). Nice symmetry of the TBM matrix $\mathbf{U}_{T ~ B M}$ inspired attempts to keep it for the description of neutrino mixing; some corrections to it were introduced [9] for better agreement with the experiments. Due to experimentally confirmed $\mathrm{CP}$ violation and nonzero value of the angle $\theta_{13}$ for neutrinos, the conceptual shift from the TBM matrix to the cobimaximal mixing with $\theta_{13} \neq 0, \theta_{23}=\frac{\pi}{4}$, $\delta_{C P}= \pm \frac{\pi}{2}$ in (2), followed (see [24-28]); proper reasons for it and justifications can be found in the above citations. The extended corresponding symmetries are now under investigations (see, for example, [29-32]) to reconstruct from them the following neutrino mixing matrix with cobimaximal mixing:

$$
\begin{aligned}
& \mathbf{U}_{C B M} \\
& \quad=\left(\begin{array}{ccc}
c_{12} c_{13} & s_{12} c_{13} & \mp i s_{13} \\
-\frac{\sqrt{2}}{2}\left(s_{12} \pm i s_{13} c_{12}\right) & \frac{\sqrt{2}}{2}\left(c_{12} \mp i s_{13} s_{12}\right) & \frac{\sqrt{2}}{2} c_{13} \\
\frac{\sqrt{2}}{2}\left(s_{12} \mp i s_{13} c_{12}\right) & -\frac{\sqrt{2}}{2}\left(c_{12} \pm i s_{13} s_{12}\right) & \frac{\sqrt{2}}{2} c_{13}
\end{array}\right) .
\end{aligned}
$$

The phenomenological description of neutrino mixing has much in common with quark mixing; the latter is described similarly to (1) by the Cabibbo-KobayashiMaskawa (CKM) matrix V. Mixing and $\mathrm{CP}$ violation for quarks are smaller than for neutrinos and the quark mixing matrix $\mathbf{V}$ is rather close to the unitary matrix. Small deviations from the unitary matrix were described, for example, by Wolfenstein parameters $\lambda, A, \rho, \eta[33]$. Looking for some more global symmetry in mixing, researchers explored parallels between neutrino and quark mixings. Also for neutrinos there has been a proposal for the deviation parameters from the unitary matrix; these parameters were expressed via the Wolfenstein parameters through empiric relations [34] and the hypothesis of quark-lepton complementarity (QLC) and of self-lepton complementarity (SC) appeared [34,35]. Quark-lepton complementarity [36-38] related the mixing angles of quarks to those of neutrinos. Relevant to it is the fact that without CP violation the real part of the mixing matrix remains and it represents the $3 \mathrm{D}$ rotation in real space.

Our analysis in what follows employs the experimental data for November 2018, which yields the following numerical Best Fit values for the mixing matrix entries [39]:

$$
\begin{aligned}
& \mathbf{U}_{\text {best fit }} \\
& =\left(\begin{array}{ccc}
0.821 & 0.551 & -0.123+0.086 i \\
-0.283+0.054 i & 0.590+0.036 i & 0.753 \\
0.490+0.046 i & -0.588+0.031 i & 0.641
\end{array}\right) .
\end{aligned}
$$

With account for $3 \sigma$ experimental error spread, the absolute values of the mixing matrix entries are currently within the following intervals [39]:

$$
\begin{aligned}
& |\mathbf{U}|_{3 \sigma} \\
& \quad=\left(\begin{array}{llll}
0.796 \leftrightarrow 0.843 & 0.518 \leftrightarrow 0.586 & 0.143 \leftrightarrow 0.156 \\
0.214 \leftrightarrow 0.533 & 0.425 \leftrightarrow 0.703 & 0.639 \leftrightarrow 0.784 \\
0.246 \leftrightarrow 0.505 & 0.451 \leftrightarrow 0.721 \quad & 0.603 \leftrightarrow 0.755
\end{array}\right) .
\end{aligned}
$$

The discussion of the ways the underlaying data sets are obtained and elaborated, as well as the account for the experimental and evaluation errors, their evaluation and relation with each other, can be found, for example, in $[13,39-42]$ and other relevant publications.

In the present work we will consider the cobimaximal matrix (CBM), fit with the existing experimental data, and we will use the Jarlskog invariant to control the degree of the $\mathrm{CP}$ violation. We will use the matrix exponential to frame the CBM ansatz in the exponential parameterization and get the value of the rotation angle in space. With the help of the exponential parameterization we will show that neutrino mixing reduces to rotations in real space and further action of the $\mathrm{CP}$ violating matrix. We will redefine the quark-lepton complementarity by including all three mixing angles in it, and demonstrate that CBM parameterization provides exact quark-lepton complementarity in this sense. 


\section{Exponential parameterization of mixing matrix}

The mixing matrix can be presented in the following exponential form:

$\mathbf{U}_{\text {exp }}=\exp \mathbf{A}$,

which was, to our best knowledge, first proposed for neutrinos in [43] and for quarks in [44]. Relevant studies of the exponential mixing matrix were conducted in [44-50]. The anti-Hermitian form of the matrix $\mathbf{A}$ ensures that the transforms by the matrix $\mathbf{U}_{\text {exp }}$ are unitary. The exponential parameterization (7) has several advantages; one of them consists in that the mixing can be presented as the product of matrices in charge of the real rotation, $\mathbf{P}_{\mathrm{Rot}}=e^{\mathbf{A}_{\mathrm{Rot}}}$, and the $\mathrm{CP}$ violation, $\mathbf{P}_{C P}=e^{\mathbf{A}_{\mathbf{C P}}}$, which form new unitary parameterization

$\tilde{\mathbf{U}}=\mathbf{P}_{\text {Rot }} \mathbf{P}_{\mathrm{CP}}, \quad \mathbf{P}_{\text {Rot }}=e^{\mathbf{A}_{\text {Rot }}}, \quad \mathbf{P}_{C P}=e^{\mathbf{A}_{\mathrm{CP}}}$.

The exponentials $\mathbf{A}_{\mathrm{Rot}}$ and $\mathbf{A}_{\mathrm{CP}}$ describe the rotation and the $\mathrm{CP}$ violation respectively. Based on the experimentally verified values of the mixing matrix (5), one can obtain the entries for the matrix $\mathbf{A}$ in the exponent by taking matrix logarithm of the mixing matrix. This procedure can be executed in several different ways (see, for example, [51,52]); computer programs for analytical calculations, such as Mathematica, allow fast computations. According to the November 2018 data [39], the mixing angles values are as follows:

$\theta_{12} \cong 33.82_{-0.76^{\circ}}^{\circ+0.78^{\circ}}, \quad \theta_{23} \cong 49.6_{-1.2^{\circ}}^{\circ}$,
$\theta_{13} \cong 8.61^{\circ}{ }_{-0.13^{\circ}}, \quad 0.13^{\circ}, \quad \delta_{C P} \cong 215^{\circ}{ }_{-29^{\circ}}$.

Upon taking matrix logarithm with account for the angles (9), we get the argument of the exponent (7) in the form of the following matrix:

$\mathbf{A}=\left(\begin{array}{ccc}-0.0147 i & 0.4982+0.0323 i & -0.3661+0.0787 i \\ -0.4982+0.0323 i & 0.0292 i & 0.8018+0.0184 i \\ 0.3661+0.0787 i & -0.8018+0.0184 i & -0.0144 i\end{array}\right)$.

Let us consider the CBM matrix (4), that is the standard parameterization matrix (2) for $\theta_{23}=\pi / 4, \delta_{C P}=-\pi / 2$, for the angles $\theta_{12}=33.51^{\circ}, \theta_{13}=8.695^{\circ}$ within the experimentally allowed range. The explanation of why exactly these values $\theta_{12,23}$ are chosen among others within $3 \sigma$ spread, will be provided in the following section of this paper. Then for these angles we obtain $\mathbf{U}_{\text {best fit }}$ CBM $=\exp \left[\mathbf{A}_{C B M}\right]$ following (7), where $\mathbf{A}_{C B M}$ reads as follows:

$$
\begin{aligned}
& \mathbf{A}_{C B M} \\
& =\left(\begin{array}{ccc}
-0.0235 i & 0.5523+0.0524 i & -0.2303+0.1413 i \\
-0.5523+0.0524 i & 0.0467 i & 0.7604+0.0347 i \\
0.2303+0.1413 i & -0.7604+0.0347 i & -0.0232 i
\end{array}\right) .
\end{aligned}
$$

The resulting CBM matrix has the following entries:

$$
\begin{aligned}
& \mathbf{U}_{\text {best fit CBM }} \\
& \quad=\left(\begin{array}{ccc}
0.824 & 0.546 & 0.151 i \\
-0.390+0.089 i & 0.590+0.059 i & 0.699 \\
0.390+0.089 i & -0.590+0.059 i & 0.699
\end{array}\right) .
\end{aligned}
$$

The parameterization of the mixing matrix (1) in the exponential form (7) $\mathbf{U}_{\exp }=\exp \mathbf{A}$, where $\mathbf{A}$ is given by (10), exactly reproduces the experimental data (5). Moreover, the $\mathrm{CBM}$ matrix (12) $\mathbf{U}_{\text {best fit }} \mathrm{CBM}=\exp \left[\mathbf{A}_{C B M}\right]$, where $\mathbf{A}_{C B M}$ is given by (11), is within the allowance (6), determined by the experimental data. Matrix $\mathbf{A}$ has zero trace $\operatorname{Tr} \mathbf{A}=0$ (same for $\mathbf{A}_{C B M}$ etc). Apart small diagonal imaginary elements of the matrices $\mathbf{A}$ (10) (and $\mathbf{A}_{C B M}$ (11))

$$
\begin{aligned}
& \mathbf{A}_{\text {diag }}=i \operatorname{diag}\left\{\alpha_{1}, \alpha_{2}, \alpha_{3}\right\}, \\
& \alpha_{1} \cong \alpha_{3} \cong-\alpha_{2} / 2, \quad \alpha_{2}=0.0292 .
\end{aligned}
$$

we can distinguish the matrices of pure rotation and $\mathrm{CP}$ violation

$\mathbf{A}=\mathbf{A}_{1}+\mathbf{A}_{\text {diag }} \cong \mathbf{A}_{1}=\mathbf{A}_{\text {Rot }}+\mathbf{A}_{C P}$,

where the matrix

$\mathbf{A}_{\text {Rot }}=\operatorname{Re}[\mathbf{A}]=\left(\begin{array}{ccc}0 & 0.4982 & -0.3661 \\ -0.4982 & 0 & 0.8018 \\ 0.3661 & -0.8018 & 0\end{array}\right)$

describes the rotations in 3D space and the identity $\mathbf{P}_{\mathrm{Rot}}$. $\left[\mathbf{P}_{\text {Rot }}\right]^{\text {Transposed }}=\mathbf{I}=\operatorname{diag}\{1,1,1\}$ holds. The other matrix in (15) describes the $\mathrm{CP}$ violation:

$$
\begin{aligned}
\mathbf{A}_{C P} & =i \operatorname{Im}\left[\mathbf{A}-\mathbf{A}_{\mathrm{diag}}\right] \\
& =\left(\begin{array}{ccc}
0 & 0.0323 i & 0.0787 i \\
0.0323 i & 0 & 0.0184 i \\
0.0787 i & 0.0184 i & 0
\end{array}\right) .
\end{aligned}
$$

In this way we separate the real rotational matrix, $\mathbf{P}_{\text {Rot }}=$ $e^{\mathbf{A}_{\text {Rot }}}$, and the term $\mathbf{P}_{C P}=e^{\mathbf{A}_{\mathbf{C P}}}$, which describes the $\mathrm{CP}$ violation. Note, that the above proposed way to distinguish $\mathbf{A}_{\mathrm{CP}}$ and $\mathbf{A}_{\mathrm{Rot}}$ is not the only one. A variety of matrices for real rotation and $\mathrm{CP}$ violation can be distinguished, which yield the matrix $\tilde{\mathbf{U}}=\mathbf{P}_{\mathrm{Rot}} \mathbf{P}_{\mathrm{CP}}$. The explicit expression for the latter in our case reads as follows:

$\tilde{\mathbf{U}}=\left(\begin{array}{ccc}0.8213+0.008068 i & 0.5511+0.024291 i & -0.12401+0.07493 i \\ -0.28228+0.07839 i & 0.5901+0.004751 i & 0.7522-0.01136 i \\ 0.4884+0.03162 i & -0.5888+0.027606 i & 0.6421+0.02769 i\end{array}\right)$.

The absolute values of the entries of the matrix $\tilde{\mathbf{U}}$ (18) agree quite well with those determined from the experimental values for neutrino mixing $\mathbf{U}_{\text {bestfit }}(5)$ and they are within the range $3 \sigma$ (6); the entries of $\tilde{\mathbf{U}}$ differ from those of $\mathbf{U}_{\text {bestfit }}$ (5) 
by small imaginary parts of the order $10^{-2}$, which is within the order of the accuracy of $\mathbf{U}$.

In complete analogy we can treat the exponential form of the CBM matrix to distinguish in (11) the rotational component

$\mathbf{A}_{\text {Rot CBM }}=\left(\begin{array}{ccc}0 & 0.5523 & -0.2303 \\ -0.5523 & 0 & 0.7604 \\ 0.2303 & -0.7604 & 0\end{array}\right)$,

and the $\mathrm{CP}$ violating component

$\mathbf{A}_{C P \text { CBM }}=\left(\begin{array}{ccc}0 & 0.0524 i & 0.1413 i \\ 0.0524 i & 0 & 0.0348 i \\ 0.1413 i & 0.0348 i & 0\end{array}\right)$.

The imaginary diagonal for the CBM matrix reads as follows:

$\mathbf{A}_{\text {diag }}^{C B M}=i \operatorname{diag}\{-0.0235,0.0468,-0.0232\}$.

Note, that the deviation from exact equality in (14) is very small: $<1 \%$ and it holds better for the recent data [39] than for the old data 2016 [41]. Imaginary diagonal matrix $\mathbf{A}_{\text {diag }}$ yields the complex diagonal exponential matrix

$\mathbf{P}_{\text {diag }}=\exp \left[\mathbf{A}_{\text {diag }}\right]=\operatorname{diag}\left\{e^{i \alpha_{1}}, e^{i \alpha_{2}}, e^{i \alpha_{3}}\right\}$.

The matrix $\mathbf{A}_{1}=\mathbf{A}_{\text {Rot }}+\mathbf{A}_{C P}$ (15) is the sum of the rotational term $\mathbf{A}_{\text {Rot }}$ (16) and the $C P$-violating term $\mathbf{A}_{C P}$ (17). For the recently established most probable value of the $\mathrm{CP}$ violating phase $\delta_{C P}=215^{\circ}$ we therefore get

$$
\begin{aligned}
& \mathbf{A} \cong \mathbf{A}_{1}=\mathbf{A}_{\mathrm{Rot}}+\mathbf{A}_{\mathrm{CP}}\left(\begin{array}{ccc}
0 & c_{2} e^{i \delta_{2}} & -c_{1} e^{i \delta_{1}} \\
-c_{2} e^{-i \delta_{2}} & 0 & c_{3} e^{i \delta_{3}} \\
c_{1} e^{-i \delta_{1}} & -c_{3} e^{-i \delta_{3}} & 0
\end{array}\right), \\
& \delta_{1}=168^{\circ}, c_{1}=-0.374, \\
& \delta_{2}=4^{\circ}, \quad c_{2}=0.499, \\
& \delta_{3}=1^{\circ}, \quad c_{3}=0.802 .
\end{aligned}
$$

For CBM matrix, where $\delta_{C P}=270^{\circ}$, we obtain

$$
\begin{aligned}
\mathbf{A}_{1}^{C B M} & =\mathbf{A}_{\mathrm{Rot}}^{C B M}+\mathbf{A}_{\mathrm{CP}}^{C B M} \\
& =\left(\begin{array}{ccc}
0 & 0.5548 e^{i 5^{\circ}} & 0.2702 e^{i 148^{\circ}} \\
-0.5548 e^{-i 5^{\circ}} & 0 & 0.7612 e^{i 3^{\circ}} \\
-0.2702 e^{-i 148^{\circ}} & -0.7612 e^{-i 3^{\circ}} & 0
\end{array}\right) .
\end{aligned}
$$

Both (23) and (24) satisfy the spread $3 \sigma$. The absolute values of the entries of $\mathbf{A}_{1}$ matrix (23) for the experimental spread $3 \sigma$ [see (4)] are as follows:

$$
\left|\mathbf{A}_{1}\right|_{3 \sigma}=\left(\begin{array}{ccc}
0 & 0.439 \leftrightarrow 0.660 & 0.047 \leftrightarrow 0.428 \\
0.439 \leftrightarrow 0.660 & 0 & 0.628 \leftrightarrow 0.929 \\
0.047 \leftrightarrow 0.428 & 0.628 \leftrightarrow 0.929 & 0
\end{array}\right) .
$$

The anti-Hermitian form of the matrix in the exponential ensures unitarity of the proper transform: $\mathbf{U}_{\exp }^{-1} \cdot \mathbf{U}_{\exp }=\mathbf{U}_{\text {exp }}^{+}$.
$\mathbf{U}_{\text {exp }}=\mathbf{I}$. Other than (7) exponential forms for the mixing matrix can be constructed. For example, the following one can be considered:

$\mathbf{U}=\exp i \theta \mathbf{H}$,

where $\mathbf{H}$ is the traceless $(3 \times 3)$ Hermitian matrix with the normalization $\operatorname{tr}\left[\mathbf{H}^{2}\right]=2$, which formally frames neutrinos into SU(3) group. Although at this moment of time there are no obvious physical reasons to frame neutrinos in SU(3) group, the underlying mathematics determines the specific way to distinguish the group parameter $\theta$ in (26):

$\theta=\left(-\operatorname{tr}\left[\mathbf{A}^{2} / 2\right]\right)^{1 / 2}$,

and links it with the presentation of neutrino mixing in terms of rotation and $\mathrm{CP}$ violation, which we will consider in what follows. Omitting small diagonal part $\mathbf{A}_{\text {diag }}$, we get $\theta \cong \sqrt{c_{1}^{2}+c_{2}^{2}+c_{3}^{2}}$ written in terms of $c_{1,2,3}$ defined in (23). Moreover, following [53], we get for the mixing matrix the following explicit form:

$$
\begin{aligned}
\mathbf{U}= & \exp i \theta \mathbf{H} \\
= & \sum_{k=0,1,2}\left(\frac{e^{\frac{2}{\sqrt{3}} i \theta \sin (\phi+2 \pi k / 3)}}{1-2 \cos (2(\phi+2 \pi k / 3))}\right. \\
& \times\left[\mathbf{H}^{2}+\mathbf{H} \frac{2 \sin (\phi+2 \pi k / 3)}{\sqrt{3}}\right. \\
& \left.\left.-\mathbf{I} \frac{1+2 \cos (2(\phi+2 \pi k / 3))}{3}\right]\right),
\end{aligned}
$$

where the angle $\phi$ is expressed via $\operatorname{det} \mathbf{H}$ as follows [53]:

$\phi=\frac{1}{3}\left(\arccos \left(\frac{3}{2} \sqrt{3} \operatorname{det} \mathbf{H}\right)-\frac{\pi}{2}\right)$.

The above formulation in the framework of the exponential ansatz is valid for SU(3) group. This approach is different from that applied for the study of mixing in [54], where the Cayley-Hamilton theorem [55] for the exponential matrix was employed:

$\exp \mathbf{A}=a_{0} \mathbf{I}+a_{1} \mathbf{A}+a_{2} \mathbf{A}^{2}$,

and $a_{0,1,2}$ were computed, following the method of [55].

By elaborating the last experimental data with the help of the Mathematica program, we obtain the following explicit matrix form for $\mathbf{H}$ :

$\mathbf{H}_{\text {Nov }}^{2018} \cong\left(\begin{array}{ccc}-0.014 & 0.032-0.490 i & 0.077+0.360 i \\ 0.032+0.490 i & 0.029 & 0.018-0.789 i \\ 0.077-0.360 i & 0.018+0.789 i & -0.014\end{array}\right)$.

High precision computations show that the matrix $\mathbf{H}$ is traceless and $\operatorname{det}(\mathbf{H})=-0.076$, the latter being the invariant for 
the $\mathrm{SU}(3)$ group. For the CBM parameterization, fitted with the available experimental data [see matrix (12)], we get the following matrix $\mathbf{H}_{C B M}$ :

$$
\mathbf{H}_{C B M}=\left(\begin{array}{ccc}
-0.0240 & 0.053-0.563 i & 0.144+0.235 i \\
0.053+0.563 i & 0.048 & 0.035-0.775 i \\
0.144-0.235 i & 0.035+0.775 i & -0.024
\end{array}\right) .
$$

Based on the available experimental data, we fitted (26) and obtained $\theta$ and $\phi$ for some sets of measurements; we found that they varied for different from each other sets of measurements. Based on the data for January of 2018 [40] we get

$\theta_{v 2018}^{\operatorname{Jan}}=56.75_{-7.23^{\circ}}+3.26^{\circ}, \quad \phi_{v J a n}^{2018}=5.49_{-5.49^{\circ}}+4.36^{\circ}$,

and with the data for November 2018 [39] we obtain

$\theta_{v 2018}^{N o v}=58.24_{-8.62^{\circ}}+7.25^{\circ}, \quad \phi_{v N o v}^{2018}=3.78_{-10.03^{\circ}}{ }_{-3.94^{\circ}}$.

For the CBM parameterization (12) consistent with the most recent experimental data [39], we get

$\theta_{C B M}=56.19^{\circ}, \quad \phi_{v}^{C B M}=6.88^{\circ}$.

The respective values for quarks are significantly smaller:

$\theta_{q}=13.23_{-0.02^{\circ}}^{\circ+0.02^{\circ}}, \quad \phi_{q}=-0.25^{\circ}{ }_{-0.01^{\circ}}^{\circ}$.

Importantly, the angle $\theta$ - the parameter (27) in SU(3) group formalism - is very close to the angle of 3D rotation $\Phi$ in the rotation matrix $\mathbf{P}_{\text {Rot }}$, which will be distinguished and used for the study of QLC in Sect. 4. Slight difference between $\theta$ and $\Phi$ is due to the way the rotation matrix $\mathbf{P}_{\text {Rot }}$ is distinguished as $\operatorname{Re}[\mathbf{A}]$ and not $\left|\mathbf{A}_{1}\right|$. The behavior of the angles $\theta$ and $\phi$ in the exponential presentation (26)-(29) will be further studied in Sect. 5. As regards the exponential matrix $\mathbf{A}(23)$ in (7), note that the angle $\theta$ is by definition (27) independent on the phases $\delta_{1,2,3}$ in (23).

\section{Jarlskog invariant}

Due to the unitarity of the mixing matrix the following relations hold:

$$
\sum_{\alpha=e, \mu, \tau} U_{\alpha i} U_{\alpha j}^{*}=\delta_{i j}, \quad \sum_{i=1,2,3} U_{\alpha i} U_{\beta i}^{*}=\delta_{\alpha \beta},
$$

where $U_{\alpha i} \equiv\left\langle v_{\alpha} \mid v_{i}\right\rangle, \alpha=e, \mu, \tau, i=1,2,3$; (37) can be geometrically viewed as triangles on the complex plane, like that shown in Fig. 1.

The square of the triangle in Fig. 1 equals a half of the absolute value of the vector product of the vectors $\{\operatorname{Re} a, \operatorname{Im} a, 0\}$ and $\{\operatorname{Re} b, \operatorname{Im} b, 0\}$ :

$$
\begin{aligned}
S & =\frac{1}{2}|(\operatorname{Re} a)(\operatorname{Im} b)-(\operatorname{Re} b)(\operatorname{Im} a)| \\
& =\frac{1}{2}\left|\operatorname{Im}\left(a b^{*}\right)\right|=\frac{1}{2}\left|\operatorname{Im}\left(a^{*} b\right)\right| .
\end{aligned}
$$

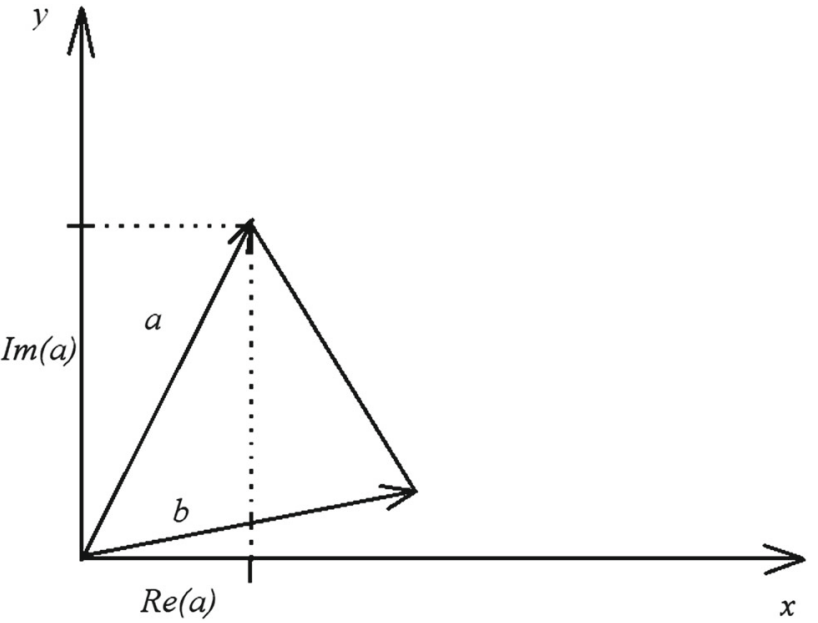

Fig. 1 Jarlskog relation presented as triangle on a complex plane in 3D space

For $a=\mathbf{U}_{e 1} \mathbf{U}_{e 3}^{*}$ and $b=-\mathbf{U}_{\mu 1} \mathbf{U}_{\mu 3}^{*}$ we get $S=$ $\frac{1}{2}\left|\operatorname{Im}\left(\mathbf{U}_{e 1} \mathbf{U}_{e 3}^{*} \mathbf{U}_{\mu 1}^{*} \mathbf{U}_{\mu 3}\right)\right|$. Due to the unitarity of the matrix $\mathbf{U}$ the following equality holds: $\mathbf{U}_{e 3}^{*} \mathbf{U}_{\mu 3}=-\mathbf{U}_{e 1}^{*} \mathbf{U}_{\mu 1}-$ $\mathbf{U}_{e 2}^{*} \mathbf{U}_{\mu 2}$, which means that the square of the triangle is $S=\frac{1}{2}\left|\operatorname{Im}\left(\mathbf{U}_{e 1} \mathbf{U}_{\mu 2} \mathbf{U}_{e 2}^{*} \mathbf{U}_{\mu 1}^{*}\right)\right|=\frac{1}{2} J$. The squares of other triangles can be calculated in similarly; they also equal $J / 2$, where $J$ is the Jarlskog invariant [56]. For the matrix $\mathbf{U}$ (2) we therefore get the Jarlskog invariant in the following explicit form:

$$
\begin{aligned}
J= & \operatorname{Im}\left(\mathbf{U}_{e 1} \mathbf{U}_{\mu 2} \mathbf{U}_{e 2}^{*} \mathbf{U}_{\mu 1}^{*}\right)=\cos \theta_{12} \sin \theta_{12} \cos ^{2} \theta_{13} \\
& \times \sin \theta_{13} \cos \theta_{23} \sin \theta_{23} \sin \delta_{C P} .
\end{aligned}
$$

Since $J \propto \sin \delta_{C P}$, it has nonzero value only in the presence of the $\mathrm{CP}$ violation. In fact, the Jarlskog invariant represents the degree of the $\mathrm{CP}$ violation, which is independent on the parameterization of the mixing matrix. The dependence $|J|$ on $\delta_{C P}$ in the standard parameterization with account for the experimental data [39] is shown in Fig. 2.

We chose $\theta_{23}$ and $\delta_{C P}$ as the arguments of $|J|$ in Fig. 2, because they are less well determined than $\theta_{12,13}$. The range of their values in Fig. 2 is chosen within the experimental spread $3 \sigma$ [see (6)]. With the data [39] we obtain the Jarlskog invariant value for neutrinos $J_{v}=-0.019_{-0.016}^{+0.046}$. The $\mathrm{CBM}$ parameterization assumes $\delta_{C P}=270^{\circ}$; the dependence $J\left(\theta_{12}, \theta_{13}\right)$ is shown in this case in Fig. 3.

For quarks the degree of the $\mathrm{CP}$ violation is about of three orders of magnitude less than for neutrinos. According to PDG [42] the value of the Jarlskog invariant for quarks is $J_{q}=(3.18 \pm 0.15) \times 10^{-5}$. The dependence $J\left(\delta_{C P}, \theta_{23}\right)$ for quarks is shown in Fig. 4.

In Fig. 5 we present the dependence of the absolute value of the Jarlskog invariant $|J|$ for neutrinos on $\theta_{12}$ and $\theta_{13}$ in 


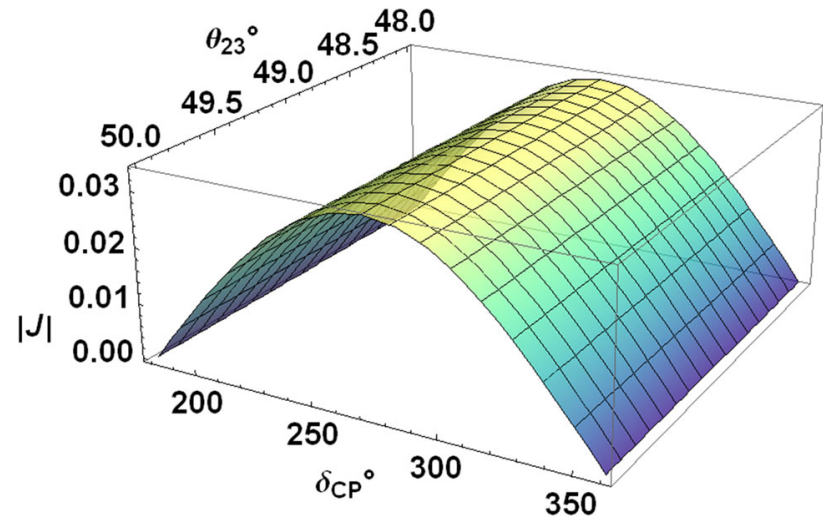

Fig. 2 Dependence of the absolute value of the Jarlskog invariant $J$ on $\theta_{23}$ and $\delta_{C P}$ for neutrinos in the standard parameterization with account for the experimental data [39]

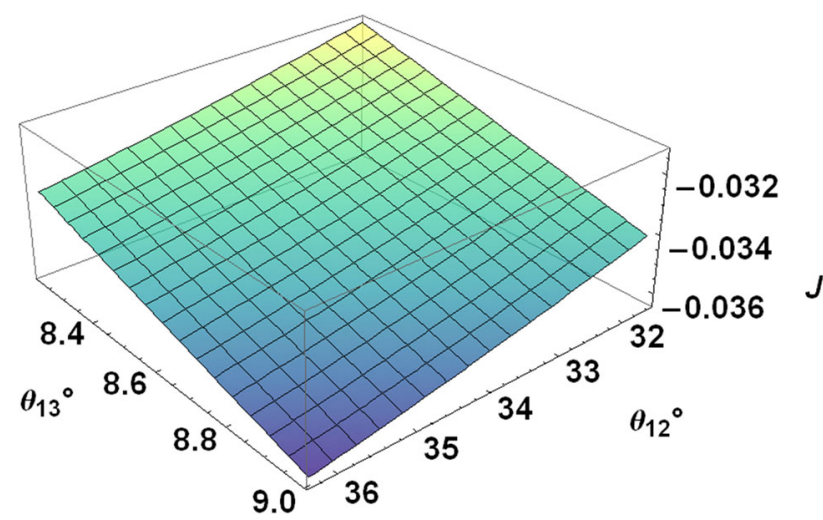

Fig. 3 The Jarlskog invariant $J$ for neutrinos in the framework of CBM parameterization as a function of the mixing angles $\theta_{12}, \theta_{13}\left(\delta_{C P}=\right.$ $\left.270^{\circ}\right)$

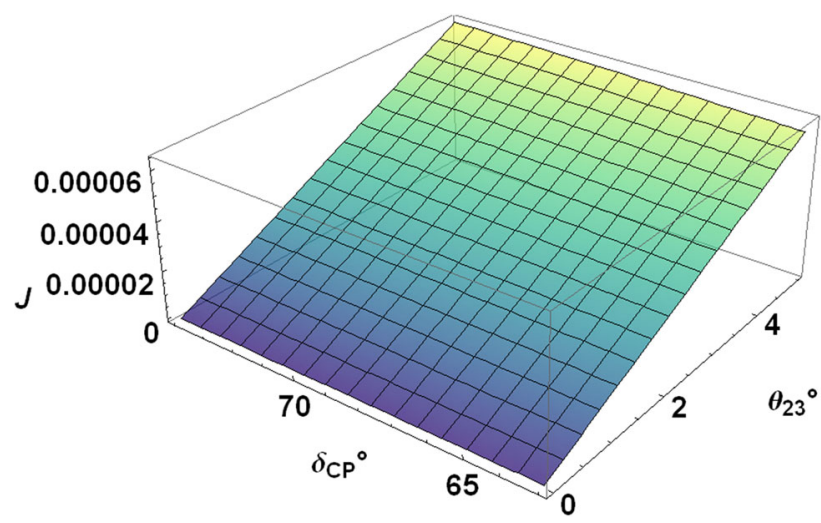

Fig. 4 Dependence of the Jarlskog invariant $J$ on $\theta_{23}$ and $\delta_{C P}$ for quarks

the CBM parameterization (4) $\left(\theta_{23}=\pi / 4, \delta_{C P}=-\pi / 2\right)$. The plot in Fig. 5 corrects the erroneous result in [51].

Let us now fix some value, $J_{f i x}$, of the Jarlskog invariant for the CBM parameterization. This yields the following analytical relation between $\theta_{12}$ and $\theta_{13}$ :

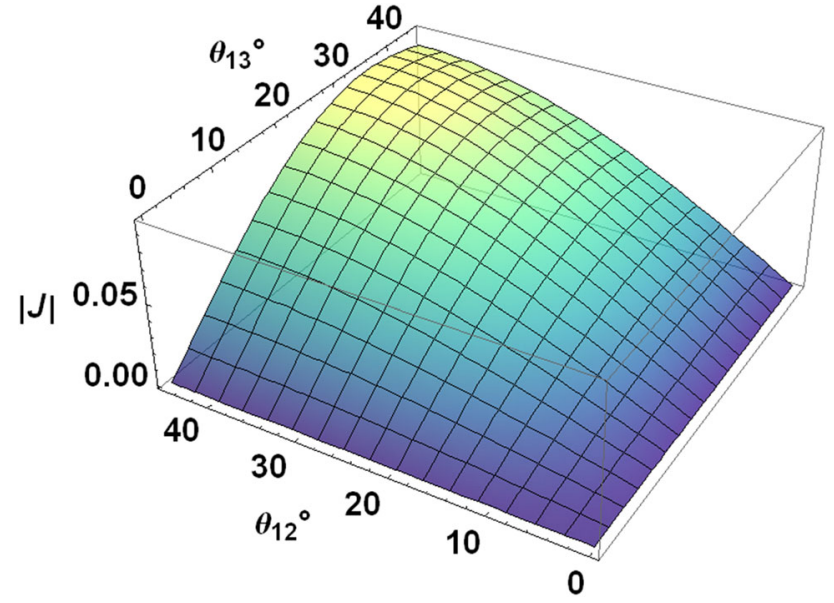

Fig. 5 Dependence of the absolute value of the Jarlskog invariant $J$ on $\theta_{12}$ and $\theta_{13}$ for neutrinos in CBM parameterization (4)

$\theta_{12}=\frac{1}{2} \arcsin \left(\frac{-4 J_{\text {fix }}}{\cos ^{2} \theta_{13} \sin \theta_{13}}\right)$.

From this relation we find $\theta_{12}=33.51^{\circ}, \theta_{13}=8.695^{\circ}$ for $J_{\text {fix }}=-0.034$ within the experimental spread $3 \sigma$; it yields the CBM matrix in the form (12) in best agreement with the available the experimental data. Thus, we have got the angles $\theta_{12}$ and $\theta_{13}$ for the CBM parameterization, where $\theta_{23}=\pi / 4$, $\delta_{C P}=-\pi / 2$, such, that the CBM parameterization fits the experimental data $|\mathbf{U}|_{3 \sigma}$ (6) and so does the Jarlskog invariant. In what follows we will address the complementarity hypothesis for quarks and neutrinos in the CBM and in the exponential parameterizations, fitted with the experimental data.

\section{Complementarity of neutrino and quark mixing}

The quark-neutrino complementarity (QLC) hypothesis was expressed in [35,36,57]; it is usually understood that the sum of the Cabibbo angle $\theta_{12}$ for quarks and of the solar mixing angle $\theta_{12}$ for neutrinos gives $\pi / 4$. Its extended formulation includes also "weak complementarity" relation for $\theta_{23}: \theta_{23 v}+$ $\theta_{23 q} \cong 45^{\circ}$ [58-61]. It would be natural to assume that the third QLC relation holds also for $\theta_{13}$. However, it appears that $\theta_{13 v}+\theta_{13 q}<10^{\circ}$, although the angles $\theta_{13}$ are established with less precision that $\theta_{12}, \theta_{23}$. Despite the third possible QLC relation in the above formulation is not realized at all appearance, we have obtained another, more general relation for neutrinos and quarks, which involves all three mixing angles, $\theta_{12}, \theta_{23}$ and $\theta_{13}$; it yields $45^{\circ}$ angle for the rotation axes of neutrinos and quarks. This $45^{\circ}$ space angle holds remarkably well through the years with different from each other data sets. We will demonstrate it in what follows.

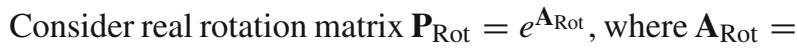
$\operatorname{Re}[\mathbf{A}]$ (16). This rotation can be presented as a turn in the 
angle $\Phi$ in $3 \mathrm{D}$ space around a definite axis $\vec{n}$, and it has the following form:

$$
\begin{aligned}
\mathbf{P}_{\mathrm{Rot}} & =e^{\mathbf{A}_{\mathrm{Rot}}}, \\
\mathbf{A}_{\mathrm{Rot}} & =\left(\begin{array}{ccc}
0 & \lambda & -\mu \\
-\lambda & 0 & v \\
\mu & -v & 0
\end{array}\right)=\Phi\left(\begin{array}{ccc}
0 & -n_{z} & n_{y} \\
n_{z} & 0 & -n_{x} \\
-n_{y} & n_{x} & 0
\end{array}\right) .
\end{aligned}
$$

Note that $\mathbf{A}_{\text {Rot }}$ in (41) is close to, but different from $\left|\mathbf{A}_{1}\right|$ in (23). It is easy to calculate the rotation angle $\Phi=$ $\pm \sqrt{\lambda^{2}+\mu^{2}+v^{2}} \cong \pm \theta$ [see (27)], where both signs are possible, and the coordinates of the rotation vector are $\vec{n}=$ $\left(-\frac{v}{\Phi},-\frac{\mu}{\Phi},-\frac{\lambda}{\Phi}\right)$. For certainty we choose the sign + and, for example, for tribimaximal matrix (TBM) we get

$\vec{n}_{\mathrm{TBM}}=-(0.7858,0.2235,0.5777), \quad \Phi_{\mathrm{TBM}} \cong 56.6^{\circ}$.

The coordinates of $\vec{n}_{v}$ in the standard and other parameterizations vary, dependently on the experimental data set. In 2016 they were as follows:

$$
\begin{aligned}
& \vec{n}_{v}^{2016}=-(0.8094,0.2417,0.5353), \\
& \Phi_{v}^{2016} \cong 59.06^{\circ}+4.36^{\circ}
\end{aligned}
$$

The January 2018 data yields

$$
\begin{aligned}
& \vec{n}_{v}^{\text {Jan2018 }}=-(0.7859,0.3272,0.5246), \\
& \Phi_{v}^{\text {Jan } 2018} \cong 56.28^{\circ}+4.58^{\circ}
\end{aligned}
$$

based on November 2018 data [39], we get

$$
\begin{aligned}
\vec{n}_{v}^{\text {Nov } 2018} & =-(0.7919,0.3616,0.492), \\
\Phi_{v}^{\text {Nov2018 }} \cong 58.01^{\circ}+7.07^{\circ} &
\end{aligned}
$$

For quarks, based on 2017 data [42], we obtain the following coordinates and the angle:

$$
\begin{aligned}
& \vec{n}_{q}^{2017}=-(0.1810,0.0141,0.9834), \\
& \Phi_{q}^{2017} \cong 13.23^{\circ}{ }_{-0.03^{\circ}}+0.00^{\circ}
\end{aligned}
$$

For CBM parameterization we get with account for the matrices (11), (12), which are consistent with the experimental spread $3 \sigma$, the following coordinates and angle:

$\vec{n}_{\mathrm{CBM}}=-(0.7858,0.2380,0.5708), \quad \Phi_{\mathrm{CBM}} \cong 55.4^{\circ}$.

Now upon the comparison of the values for neutrinos (42)(45) with those for quarks (46), we note that the rotation axes for neutrinos and quarks constitute with each other the angles $\approx 44^{\circ}-50^{\circ}$ and these angles varies in fairly small range around $45^{\circ}$, dependently on the experimental data set. Moreover, the angle between the rotation vector (47) in the CBM parameterization $\mathbf{U}_{\text {best fit }}$ CBM (12), consistent with current experimental data, and the vector (46) for quarks, is exactly

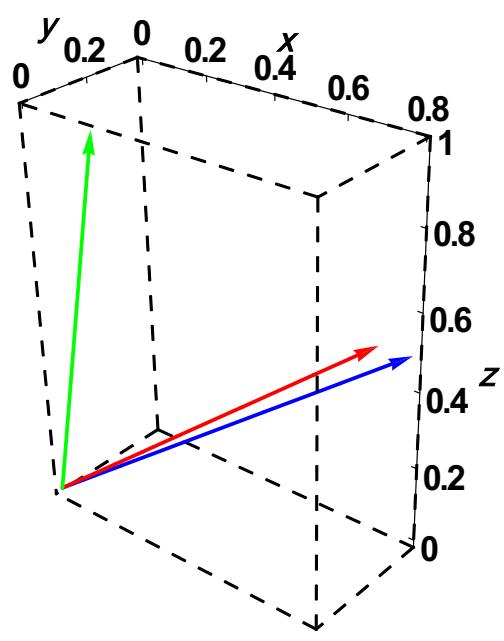

Fig. 6 Axes of rotation in real space: for quarks - green arrow, for neutrinos Best Fit 2018 - blue arrow, for neutrinos in CBM parameterization with $3 \sigma$ spread of Best Fit 2018 - red arrow

$45.0^{\circ}$. So, the angle between the quark and neutrino rotation axes is relatively stable, $\approx 45^{\circ}$, despite the values of $\vec{n}$ и $\Phi$ vary from year to year. For the CBM parameterization this angle $=45.0^{\circ}$ (see Fig. 6 , where all the axes are drawn with the opposite signs $\vec{n} \rightarrow-\vec{n}$ to show them in first quadrants for clarity); this means exact complementarity of mixing for neutrinos and quarks in CBM parameterization, based on the data for all three mixing angles. This fact in turn makes us look deeper for the underlying symmetries.

\section{Study of the CP-violation in the exponential parameterization}

With the last experimental data November 2018 [39] we obtained the matrix $\mathbf{A}_{1}$ (23) of the exponential parameterization, where $\delta_{1}=168^{\circ}, \delta_{2}=4^{\circ}, \delta_{3}=1^{\circ}$. We have also obtained the dependences of the angles $\theta \approx \Phi$ and $\phi$ for neutrinos on the CP-violating phase $\delta_{C P}$ in the standard parameterization; they are shown it in Fig. 7.

The plots in Fig. 7 demonstrate that in the likely range of values $\delta_{C P} \sim\left[180^{\circ}-360^{\circ}\right]$ the angle $\theta \approx \Phi$ (27) for space rotations for neutrinos is in narrow range $\left[56^{\circ}-60^{\circ}\right]$. The angle $\phi$ is at its maximum for $\delta_{C P}=270^{\circ}$.

The relation between $\delta_{1,2,3}$ values [see matrix $\mathbf{A}_{1}$ (23)] and the CP violating phase $\delta_{C P}$ is demonstrated in Fig. 8 . The analysis of the behavior of the function $\delta_{1}\left(\delta_{C P}\right)$ in the left plot in Fig. 8 shows that its minimal value $\delta_{1}^{\min }=147.2^{\circ}$ is reached for $\delta_{C P}=302^{\circ}$. The phase $\delta_{C P}=270^{\circ}$ corresponds $\delta_{1} \cong 151.6^{\circ}$ and currently likely value $\delta_{C P}=215^{\circ}$ corresponds $\delta_{1}=167.9^{\circ}$. Due to large uncertainty for $\delta_{C P}$, the range for $\delta_{1}$ is $\approx\left[145^{\circ}-180^{\circ}\right]$ (see Fig. 8). The behaviors $\delta_{2}\left(\delta_{C P}\right)$ and $\delta_{3}\left(\delta_{C P}\right)$ are qualitatively similar each other (see 
Fig. 7 Dependence of $\theta\left(\delta_{C P}\right)$ - left plot, $\varphi\left(\delta_{C P}\right)$ - right plot
Fig. 8 Dependence $\delta_{1}\left(\delta_{C P}\right)-$ left plot; $\delta_{2}\left(\delta_{C P}\right)$ - right plot, upper line, $\delta_{3}\left(\delta_{C P}\right)$ - right plot, lower line
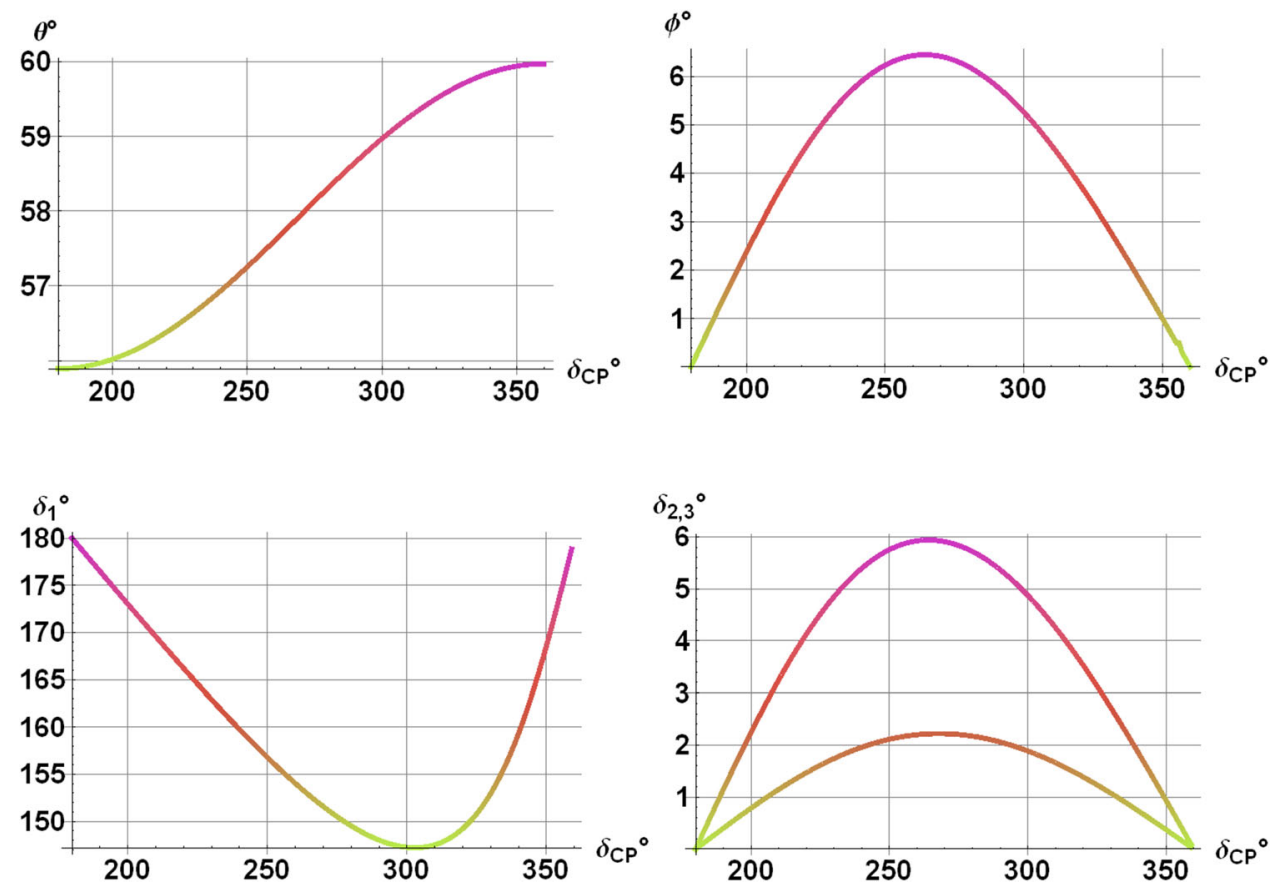

Fig. 8); they have their maximums at $\delta_{C P} \approx 270^{\circ}$, but the range of values for $\delta_{2}$ is $\sim 3$ times more than that for $\delta_{3}$ (see Fig. 8).

Now let us consider the contribution of the small imaginary diagonal matrix $\mathbf{A}_{\text {diag }}=i \operatorname{diag}\left\{\alpha_{1}, \alpha_{2}, \alpha_{3}\right\}$ of the exponential parameterization (7) of the PMNS matrix $\mathbf{U}$ to the CP violation with the help of the Jarlskog invariant $J$. The matrix $\mathbf{A}_{\text {diag }}$ apparently reminds the Majorano term in the neutrino mixing, beyond the scope of the present paper; $\mathbf{A}_{\text {diag }}$ comes due to the CP violation. It follows from (10) that the entries of $\mathbf{A}_{\text {diag }}$ obey with precision relation (14): $\alpha_{1} \cong \alpha_{3} \cong-\alpha_{2} / 2$. Let us then consider the following parameterization for $\mathbf{A}_{\text {diag }}$ :

$\mathbf{A}_{\text {diag }} \cong i \operatorname{diag}\{-\alpha, 2 \alpha,-\alpha\}$.

Figure 9 shows the behavior of the Jarlskog invariant $J$ as the function of the parameter $\alpha$ in (48), where $\alpha$ varies in the interval $\left[0, \alpha_{2}\right]$.

The dependence $J(\alpha)$ is linear. Moreover, it follows from Fig. 9 that even if we assume $\mathbf{A}_{\text {diag }}=0$, then the absolute value of the Jarlskog invariant changes insignificantly, from 0.01914 to 0.01875 . Thus, it is not $\mathbf{A}_{\text {diag }}$, which determines most of the $\mathrm{CP}$ violation, but $\mathbf{A}_{\mathbf{C P}}$ matrix (17), i.e. the imaginary part of the matrix $\mathbf{A}$, except for the diagonal $\mathbf{A}_{\text {diag }}$. This justifies the approximate equality in (15). The matrices of the real rotation $\mathbf{A}_{\text {Rot }}$ (16) and of the $\mathrm{CP}$ violation $\mathbf{A}_{\mathbf{C P}}$ (17) together constitute the most important part of the mixing matrix $\mathbf{A}_{1}=\mathbf{A}_{\text {Rot }}+\mathbf{A}_{C P}$ (15). The $\mathrm{CP}$ violation is described primarily by $\delta_{1}$; small contribution comes from $\delta_{2}$; the contribution of $\delta_{3}$ in the exponential matrix (23) is negligible.

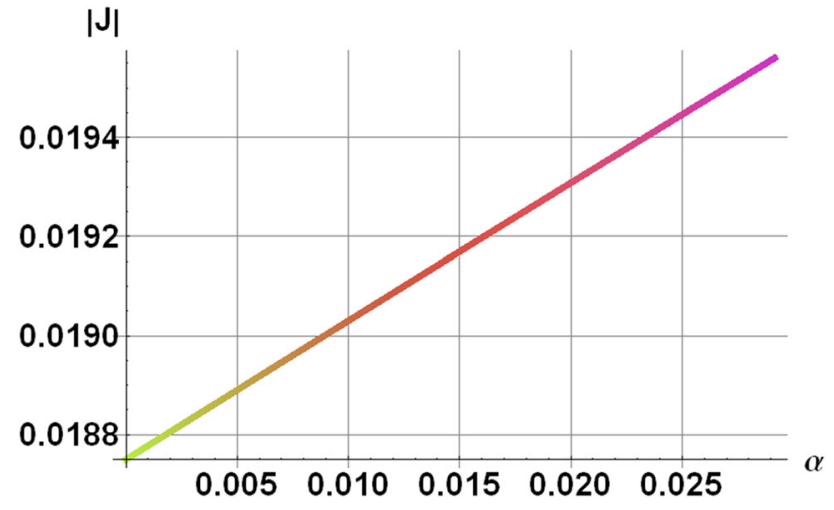

Fig. 9 Dependence of the absolute value of the Jarlskog invariant $J$ on the imaginary diagonal parameter $\alpha$ in (48)

The dependence of the absolute value of the Jarlskog invariant $|J|$ on $\delta_{1,2}$ is shown in Fig. 10. It shows the range of values for the Jarlskog invariant in the experimentally suggested range of the $\mathrm{CP}$ violation and mixing angles within the spread $3 \sigma$. If is evident (see Fig. 10) that $\delta_{1}$ primarily determines the Jarlskog invariant value; the influence of $\delta_{2}$ is small. The influence of $\delta_{3}$ is even weaker and it is omitted.

\section{Results and discussion}

With the help of the exponential form of the mixing matrix for neutrinos we analyzed the mixing data for November 2018. We compared it with that for January 2018 and for the year 2016. The exponential matrix $\mathbf{A}=\mathbf{A}_{\text {Rot }}+\mathbf{A}_{C P}+\mathbf{A}_{\text {diag }}$ allows separation of the real part $\mathbf{A}_{\text {Rot }}$ in charge of the rotation, the 


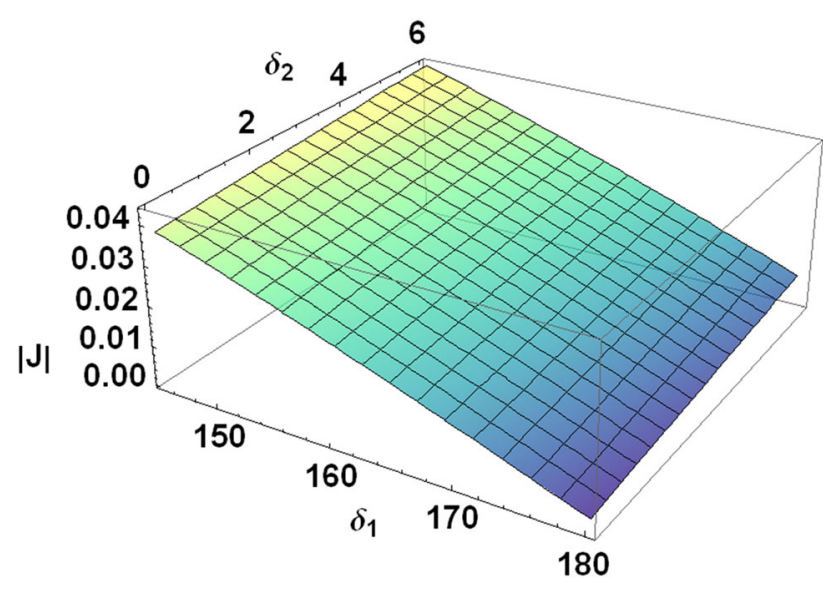

Fig. 10 Behavior of the absolute value of Jarlskog invariant $J$ as the function of $\delta_{1}$ in the range $145^{\circ}-180^{\circ}$, and $\delta_{2}$ in the range $0^{\circ}-6^{\circ}$ for $\delta_{3}=1^{\circ}$ in the exponential parameterization of the neutrino mixing matrix. The ranges are based on $3 \sigma$ spread of Best Fit

imaginary non-diagonal part $\mathbf{A}_{\mathrm{CP}}$ responsible for most of the $\mathrm{CP}$ violation, and small imaginary diagonal part $\mathbf{A}_{\text {diag }}$, whose trace is zero. We factorized in the exponential form $\mathbf{U}_{\text {exp }}=\exp \mathbf{A}$ the contributions of the rotation $\mathbf{P}_{\text {Rot }}=e^{\mathbf{A}_{\text {Rot }}}$ and of the $\mathrm{CP}$ violation $\mathbf{P}_{C P}=e^{\mathbf{A}_{C P}}$ in a new unitary matrix $\tilde{\mathbf{U}}=\mathbf{P}_{\mathrm{Rot}} \mathbf{P}_{C P}$ compliant with $3 \sigma$ spread of Best Fit. The commutators of $\mathbf{A}_{\mathrm{Rot}}, \mathbf{A}_{\mathrm{CP}}, \mathbf{A}_{\text {diag }}$ with each other are small, $\tilde{\mathbf{U}} \cong \mathbf{U}$ with the accuracy of the order $10^{-2}$. The exponential argument $\mathbf{A}_{C P}$ is imaginary and has zero diagonal entries; this matrix describes the $\mathrm{CP}$ violation quite well. Additional account for the small imaginary diagonal matrix $\mathbf{A}_{\text {diag }}=i \operatorname{diag}\left\{\alpha_{1}, \alpha_{2}, \alpha_{3}\right\}$ provides exact match with Best Fit; however, $\mathbf{A}_{\text {diag }}$ entries, $\alpha_{2} / 2 \cong-\alpha_{1} \cong-\alpha_{3} \approx 10^{-2}$, can be omitted without significant sacrifice of precision, when $\tilde{\mathbf{U}}$ is compared with Best Fit. Moreover, the latest experimental data yields the smallest ever values for the entries $\alpha_{1,2,3}$.

We compared the rotation vectors and angles for neutrinos and quarks in the exponential parameterizations fitted with the data for 2016-2018. We have found that the space angle between the rotation axes for quarks and neutrinos remains $\approx 45^{\circ}$, despite the data varied from year to year. This result has high statistical value since it involves the data for all three mixing angles and thus it can be viewed as an alternative formulation of quark-lepton complementarity hypothesis (QLC), usually based on the relations for $\theta_{12}$ and $\theta_{23}$ in its extended formulation. We have demonstrated that the cobimaximal parameterization fits the experimental range $3 \sigma$ and the angle between the rotation axes for quarks and neutrinos in the CBM parameterization equals exactly $45^{\circ}$ (see Fig. 6). Other angles in the CBM parameterization compliant with Best Fit are $\theta_{12} \cong 33.51^{\circ}, \theta_{13} \cong 8.695^{\circ}$ and it is assumed that $\theta_{23}=45^{\circ}, \delta_{C P}=-90^{\circ}$. The resulting value of the Jarlskog invariant $J$, which measures the degree of the CP violation, is $J=-0.034$, which is close to the maximal value of $\left|J_{\max }\right|=0.035$. Thus, the cobimaximal parame- terization not only fits the experimental data spread $3 \sigma$, but it provides exact complementarity for neutrinos and quarks in the above defined sense. For smaller CP violating phase, $\delta_{C P}=215^{\circ}$, we get $J_{v} \cong-0.019$ and the complementarity is satisfied approximately.

Using exponential parameterization of the neutrino mixing, we framed it into SU(3) group [see (31)-(35)] and obtained the value for the rotation angle $\theta_{v 2018}^{N o v}=$ $58.24_{-8.62^{\circ}}^{\circ}$, best fitted with the experimental data spread $3 \sigma$. This angle is very close to the rotation angle $\Phi$ around the axis $\vec{n}$ in 3D space for the rotation matrix $\mathbf{P}_{\text {Rot }}$; the small difference is due to the way this rotation matrix was distinguished. We have obtained the relations of $\theta$ and $\phi$ with the CP violating phase $\delta_{C P}$ in the standard parameterization (see Fig. 7). The angle $\theta$ by definition is independent from $\delta_{1,2,3}$ in the exponential parameterization. We have shown that in the likely wide range of values $\delta_{C P} \sim\left[180^{\circ}-360^{\circ}\right]$ the angle $\theta$ for neutrinos varies in the narrow range: $\theta \sim\left[56^{\circ}-60^{\circ}\right]$.

The correspondence between the CP violation in the standard and in the exponential parameterizations is established. For the exponential parameterization the hierarchy of the complex entries $(1,3),(2,3),(3,2)$ of the matrix exponential with the phases $\delta_{1} \approx 150^{\circ}-180^{\circ}, \delta_{2} \approx 4^{\circ}, \delta_{3} \approx 1^{\circ}$ is established.

With the help of the Jarlskog invariant we measured the $\mathrm{CP}$ violation in the framework of the standard, cobimaximal and exponential parameterizations (see Figs. 2, 3, 4, 5, $9,10)$. The major variation of the Jarlskog invariant in the exponential parameterization is induced by $\delta_{1}$. For the currently likely value $\delta_{C P} \approx 215^{\circ}$, the exponential parameter $\delta_{1}$, which mainly determines the CP violation, is in the middle of its possible range of values. The parameter $\delta_{2}$ and especially $\delta_{3}$ weakly influence the CP violation and depend on $\delta_{C P}$ in qualitatively similar to each other way (see Fig. 8).

\section{Conclusions}

1. The exponential parameterization $\mathbf{U}_{\exp }=\exp \mathbf{A}$ of the mixing matrix allows explicit factorization of pure rotation in 3D space, $\mathbf{P}_{\text {Rot }}=e^{\mathbf{A}_{\text {Rot }}}, \mathbf{A}_{\text {Rot }} \in$ Reals, and the CP violation, $\mathbf{P}_{C P}=e^{\mathbf{A}_{\mathbf{C P}}}, \mathbf{A}_{\mathrm{CP}} \in$ Imaginaries; the small diagonal elements in the latter matrix can be neglected. The result is consistent with the Best Fit data.

2. The axes of real rotation for neutrinos and quarks in the exponential parameterization constitute with each other the angle $\approx 45^{\circ}$. This result is based on the data for all three mixing angles $\theta_{12,23,13}$ and therefore it has higher statistical value than common quark-lepton complementarity (QLC). This can be viewed as an alternative formulation of QLC; it is more general than the common formulation, which involves just the angles $\theta_{12}$ and in its extended form also $\theta_{23}$. 
3. The quark-neutrino complementarity, understood in the above redefined way, holds quite well through the years 2016-2018, despite the data for the rotation angles and axes varied.

4. In the cobimaximal parameterization $(\mathrm{CBM}), \theta_{23}=\pi / 4$, $\delta_{C P}=-\pi / 2$, fitted with most recent experimental data 2018 , the angles of rotation for quarks and neutrinos constitute exactly $45^{\circ}$. Thus QLC is satisfied exactly for it.

5. In the exponential parameterization the neutrino mixing can be formally framed in SU(3) group, whose parameter $\theta$ coincides with the rotation angle $\Phi$ around the fixed axis in $3 \mathrm{D}$ space with the accuracy $0.5^{\circ}$. The angle $\theta$ does not depend on the values of $\delta_{1,2,3}$ in the exponential parameterization; dependently on the Best Fit data with $\delta_{C P}$ in the range $\left[180^{\circ}-360^{\circ}\right]$, it varies in very narrow range around $58^{\circ} \pm 2^{\circ}$.

6. Using Jarlskog invariant we have shown that in the exponential parameterization the value of $\delta_{1}$ determines most of the $\mathrm{CP}$ violation. While the $\mathrm{CP}$ violation for $v_{e}$ mixing comes from $v_{3}$ mass state, the degree of $\mathrm{CP}$ violation behaves similarly for $\nu_{\mu, \tau}$ flavor states and comes from $v_{1,2}$ mass states. This might indicate possible symmetry of the CP violation in $v_{\mu}$ and in $v_{\tau}$ neutrino mixing.

Our results demonstrate usefulness of the exponential parameterization for the analysis and interpretation of mixing. With its help we have factorized the matrices of rotation and CP violation for neutrino mixing, reformulated the hypothesis of quark-neutrino complementarity (QLC), involving the data for all three mixing angles, and shown that the CBM parameterization with arguably best symmetry in neutrino mixing exactly satisfies QLC. This induces research for new underlying physical symmetries.

Acknowledgements The authors are grateful to Prof. A. V. Borisov for useful advises and discussions.

Data Availability Statement This manuscript has no associated data or the data will not be deposited. [Authors' comment: There is no data to be deposited separately, all the needed data is present in the body of the article.]

Open Access This article is distributed under the terms of the Creative Commons Attribution 4.0 International License (http://creativecomm ons.org/licenses/by/4.0/), which permits unrestricted use, distribution, and reproduction in any medium, provided you give appropriate credit to the original author(s) and the source, provide a link to the Creative Commons license, and indicate if changes were made. Funded by SCOAP ${ }^{3}$.

\section{References}

1. S. Weinberg, Phys. Rev. Lett. 19, 1264 (1967)

2. A. Salam, Elementary Particle Theory, ed. by N. Svartholm (Almquist Forlag AB, 1968)
3. S.L. Glashow, Nucl. Phys. 22, 579 (1961)

4. B. Pontecorvo, Zh. Eksp. Teor. Fiz. 33, 549 (1957)

5. B. Pontecorvo, Zh. Eksp. Teor. Fiz. 53, 1717 (1967)

6. M. Gell-Mann, P. Ramond, R. Slansky, in Sanibel Talk, CALT-68709, February 1979, and in Supergravity (North-Holland, Amsterdam, 1979)

7. T. Fukuyama, H. Nishiura, Mass matrix of majorana neutrinos. arXiv:hep-ph/9702253

8. Z. Maki, M. Nakagawa, S. Sakata, Prog. Theor. Phys. 28, 870 (1962)

9. Y. Zhang, et al., Phys. Rev. D 86, 093019 (2012). arXiv:1211.3198 [hep-ph]

10. P.F. Harrison, D.H. Perkins, W.G. Scott, Phys. Lett. B 530, 167 (2002). arXiv:hep-ph/0202074

11. Z.-Z. Xing, Phys. Lett. B 533, 85-93 (2002). arXiv:hep-ph/0204049

12. L. Merlo, J. Phys. Conf. Ser. 335, 012049 (2011)

13. S.F. King, Models of neutrino mass, mixing and CP-violation. J. Phys. G Nucl. Part. Phys. 42, 123001 (2015). arXiv: 1510.02091

14. E. Ma, G. Rajasekaran, Phys. Rev. D 64, 113012 (2001). arXiv:hep-ph/0106291

15. K.S. Babu, E. Ma, J.W.F. Valle, Phys. Lett. B 552, 207-213 (2003). arXiv:hep-ph/0206292

16. G. Altarelli, F. Feruglio, Nucl. Phys. B 720, 64-88 (2005). arXiv:hep-ph/0504165

17. G. Altarelli, F. Feruglio, Nucl. Phys. B 741, 215-235 (2006). arXiv:hep-ph/0512103

18. A. Y. Smirnov, Talk given at DISCRETE'10-Symposium on Prospects in the Physics of Discrete Symmetries

19. F. Feruglio, C. Hagedorn, Y. Lin, L. Merlo, Nucl. Phys. B 775, 120-142 (2007). arXiv:hep-ph/0702194

20. F. Bazzocchi, L. Merlo, S. Morisi, Nucl. Phys. B 816, 204-226 (2009). arXiv:0901.2086

21. F. Bazzocchi, L. Merlo, S. Morisi, Phys. Rev. D 80, 053003 (2009). arXiv:0902.2849

22. I. de Medeiros Varzielas, G.G. Ross, Nucl. Phys. B 733, 31-47 (2006). arXiv:hep-ph/0507176

23. I. de Medeiros Varzielas, S. F. King, G.G. Ross, Phys. Lett. B 644, 153-157 (2007). arXiv:hep-ph/0512313

24. E. Ma, Phys. Rev. D 92, 051301(R) (2015)

25. E. Ma, Phys. Lett. B 752, 198-200 (2016)

26. E. Ma, G. Rajasekaran, EPL 119(3), 31001 (2017)

27. E. Ma, Phys. Lett. B 777, 332-334 (2018)

28. A. Damanik, J. Phys. Conf. Ser. 909, 012024 (2017)

29. R.N. Mohapatra, C.C. Nishi, Phys. Rev. D 86, 073007 (2012)

30. P. Chen, C.-Y. Yao, G.-J. Ding, Phys. Rev. D 92, 073002 (2015)

31. A.S. Joshipura, K.M. Patel, Phys. Lett. B 749, 159 (2015)

32. H.-J. He, W. Rodejohann, X.-J. Xu,. arXiv:1507.03541 [hep-ph]

33. L. Wolfenstein, Phys. Rev. Lett. 51(21), 1945 (1983)

34. N. Li, B.-Q. Ma, Unified parametrization of quark and lepton mixing matrices. Phys. Rev. D 71, 097301 (2005). arXiv:hep-ph/0501226

35. H. Minakata, AYu. Smirnov, Phys. Rev. D 70, 073009 (2004)

36. M. Raidal, Phys. Rev. Lett. 93, 161801 (2004)

37. X. Zhang, B.-Q. Ma, Phys. Lett. B 710, 630 (2012)

38. Y. Zhang, X. Zhang, B.-Q. Ma, Phys. Rev. D 86, 093019 (2012)

39. I. Esteban, M.C. Gonzalez-Garcia, et al., NuFIT 4.0 (2018). http:// www.nu-fit.org

40. I. Esteban, M.C. Gonzalez-Garcia, et al., NuFIT 3.2 (2018). http:// www.nu-fit.org

41. M. Gonzalez-Garcia, M. Maltoni, T. Schwetz, http://www.nu.fit. org

42. M. Tanabashi, et al. (Particle Data Group), Phys. Rev. D 98, 030001 (2018)

43. A. Strumia, F. Vissani, Neutrino masses and mixings and... (2006). arXiv:hep-ph/0606054 
44. G. Dattoli, K. Zhukovsky, Eur. Phys. J. C 50, 817 (2007)

45. G. Datolli, K.V. Zhukovsky, Eur. Phys. J. C 55, 547 (2008)

46. G. Dattoli, K. Zhukovsky, Eur. Phys. J. C 52, 591 (2007)

47. K. Zhukovsky, F. Melazzini, Eur. Phys. J. C 76, 462 (2016)

48. K. Zhukovsky, A. Borisov, Eur. Phys. J. C 76, 637 (2016)

49. K.V. Zhukovsky, Moscow Univ. Phys. Bull. 72(5), 433-440 (2017)

50. K.V. Zhukovsky, Phys. Atom. Nucl. 80(4), 690-698 (2017)

51. A. Wouk, J. Math. Anal. Appl. 11, 131 (1965)

52. T.A. Loring, Computing a logarithm of a unitary matrix with general spectrum. Numer. Linear Algebra Appl. 21, 744 (2014)

53. T.L. Curtright, C.K. Zachos, Rep. Math. Anal. Appl. 76, 401 (2015)

54. K.V. Zhukovsky, D. Dattoli, Phys. Atom. Nucl. 71(10), 1807-1812 (2008)

55. F. Ayres Jr., Schaum's Outline of Theory and Problems of Matrices (Schaum, New York, 1962), p. 181
56. C. Jarlskog, Phys. Rev. Lett. 55, 1039 (1985)

57. H. Minakata, A.Yu. Smirnov, Phys. Rev. D 70, (2004). https://doi. org/10.1103/PhysRevD.70.073009

58. F. González Canales, A. Mondragón, On quark-lepton complementarity. arXiv:hep-ph/0606175v1

59. F. Gonzalez Canales, A. Mondragon, Universal mass texture and quark-lepton complementarity. J. Phys. Conf. Ser. 171, 012063 (2009)

60. G. Sharma, B.C. Chauhan, Quark-lepton complementarity predictions for $\theta_{23}^{\mathrm{pmns}}$ and CP violation. JHEP 07, 075 (2016)

61. D. Meloni, Fron. Phys. 5, Article 43 (2017). https://doi.org/10. 3389/fphy.2017.00043 